\title{
An empirical study of the characteristics of access control model towards secure KMS in collaborative environment
}

\begin{abstract}
Knowledge Management System (KMS) is widely deployed as organizations acknowledged the importance to manage critical knowledge efficiently in secure manner. More over the community of practice $(\mathrm{CoP})$ benefit the growing of Internet by constructing a collaborative KMS for better communication. However while the excitement of expanding the KMS capabilities, some security issues exist which regard to the restriction of the access permission to knowledge, such as unauthorized access, losing and misusing critical knowledge and about other knowledge processing. Therefore, the paper reviews the criteria of access control model (ACM) because it is a competent security model to overcome the security issues by considering the characteristics of collaborative system. Consequently, this paper formulates a model of collaborative KMS access control. The proposed model can be a guidance to study further the member of ACM family particularly the role based access control (RBAC) towards secure collaborative KMS.
\end{abstract}

Keyword: Collaborative KMS; Access control model; Security; Secure KMS; RBAC 